\title{
RECENT RESULTS FROM LOW SURFACE BRIGHTNESS GALAXY SURVEYS
}

\author{
S. PHILLIPPS, J.I. DAVIES and J.A. TURNER \\ Department of Physics and Astronomy \\ University of Wales College of Cardiff \\ Wales
}

\section{Introduction}

In recent years, especially following the work of Binggeli et al. (1985) on the Virgo Cluster, there has been a great surge of interest in low surface brightness galaxies (LSBGs). One of the most important questions, of course, is - how many LSBGs are there? Here we present some results from recent surveys which have been carried out in Cardiff in an effort to shed light on this problem.

\section{Low Surface Brightness Galaxies in Clusters}

LSBGs are biased against in most 'conventional' surveys because of their low contrast against the night sky background. Such dim galaxies can only be seen if they are quite close to us, so they are swamped in size or magnitude limited 'field' samples by their more prominent cousins. We can overcome this if we concentrate on cluster galaxies, though we still need to worry about background 'interlopers'.

Previous surveys for LSBGs in the Fornax and Abell 1367 clusters (e.g. Irwin et al. 1990; Davies et al. 1989) were limited by (photographic) efficiency or (CCD) area coverage. In an attempt to keep the depth of CCD imaging, but to increase the area surveyed, we have therefore made use of the large format (1024 $\times 1024$ pixel) Thomson CCD, together with reducing optics (which result in an $\mathrm{f} / 1$ system), on the AAT. This gives a frame size of $17^{\prime} \times 17^{\prime}$ and we were able to survey a diagonal strip roughly $1.6 \times 0.2 h^{-1} \mathrm{Mpc}$ in extent (Turner et al. 1993).

By mapping and removing large scale gradients (using modified median filtering techniques) we were able to reduce 'sky' noise to $0.3 \%$ and thus set a threshold as low as $26.7 V \mu$ for our image detection. Radial intensity profiles were obtained for all candidate LSBGs and those with exponential scale length $a \geq 2$ " and central SB $S_{x} \geq 22.5 V \mu$ were accepted for our catalogue (300 objects). A restricted sample of 108 larger objects $(a \geq 3 ")$ was also considered. The LSBGs are strongly concentrated about the cluster centre, suggesting that at least one third of the full sample, and the large majority of the restricted sample, are cluster members.

The sample contains galaxies with $S_{x}$ down to $26.5 V \mu$ and with $a$ up to $14^{\prime \prime}$ or $3 h^{-1} \mathrm{kpc}$. Such LSBGs are clearly not 'dwarfs' in terms of radial scale, but have very low luminosities (of order 
$\left.10^{-3} \mathrm{~L}_{*}\right)$. The distribution of photometric parameters is consistent with earlier LSBG samples. All the accessible $\left(S_{x}, a\right)$ parameter space appears to be populated up to a maximum scale size of a few kpc, with rapidly increasing numbers of smaller objects $\left(n \propto a^{-2}\right)$, and roughly equal numbers of LSBGs in each magnitude bin in $S_{x}$ with no sign of a cut-off at the lowest level currently sampled (implying a LSBG luminosity function $n \propto L^{-1.5}$ ). We find no correlation between surface brightness and size. Thus small low surface brightness galaxies completely dominate cluster galaxy populations in terms of numbers and large low surface brightness galaxies dominate the covering factor in a cluster, but giant normal surface brightness galaxies still dominate the cluster light. The question of whether LSBGs contribute a significant mass obviously depends on their poorly known $M / L$ ratio.

If we attempt to estimate the 'total' number of large LSBGs across the whole of the currently observable SB range, roughly from 23 to $27 B \mu$ we find that there are 14 times as many LSBGs as giants in A3574, computed to be 6 times in A1367 and equal numbers in Fornax. This suggests it is the morphology of the cluster which is most important, since A3574 is a poorer cluster than A1367 but has a higher spiral fraction. Large LSBGs may therefore prefer a fairly rich but not very compact cluster.

\section{Giant LSBGs}

The most remarkable LSBG known is Malin 1 (Bothun et al. 1987). This has $a \sim 50 h^{-1} \mathrm{kpc}$ and $S_{x} \simeq 26.5 B \mu$. Other similar examples have been found but it has been hard to place any statistical constraints on their numbers. We have therefore made a deep survey specifically for Malin 1 type objects.

We have used a matched template or cross-correlation method (Phillipps \& Davies 1991) to look specifically for very LSB images with exponential radial profiles and scale sizes corresponding to Malin 1 at redshifts 0.1 to 0.3 , in very deep $V R$ band Thomson f/ 1 data (Couch et al. 1993). Sky noise is below $0.3 \%$.

The cross-correlation technique avoids the necessity for image pixels to be connected, thus allowing the opportunity for detecting very extended but very noisy images. In this sense it is a 'total' as opposed to 'isophotal' detection technique (cf. Smith et al., this meeting). Detailed simulations show that galaxies half the size of Malin 1 at $z=0.3$ and with $S_{x} 0.8 \%$ of sky $(\sim 26.8 V \mu)$ would still be picked up.

We find 19 potential Malin 1-like objects in the data. If these are real galaxies they have $S_{x} \simeq 26.5$ to $27.5 V \mu$. However, if Malin 1s were as numerous as $L_{*}$ galaxies, we should expect some 200 in our search area. We therefore conclude that giant extreme LSBGs are at least an order of magnitude less common than 'normal' galaxies of the same total luminosity. Recall, though, that such objects would necessarily have huge scale sizes - it may be that it is the huge size that is rare, not the low surface brightness.

\section{References}

Binggeli, B., Sandage, A. and Tammann, G., 1985. Astron. J., 90, 1681.

Bothun, G., Impey, C., Malin, D. and Mould, J., 1987. Astron. J., 89, 1293.

Couch, W., Jurcevic, J. and Boyle, B., 1993. Mon. Not. R. astron. Soc., 260, 241. 
Davies, J., Phillipps, S. and Disney, M., 1989. Mon. Not. R. astron. Soc., 239, 703.

Irwin, M., Davies, J., Disney, M. and Phillipps, S., 1990. Mon. Not. R. astron. Soc., 245, 289.

Phillipps, S. and Davies, J., 1991. Mon. Not. R. astron. Soc.., 251, 105.

Turner, J., Phillipps, S., Davies, J. and Disney, M., 1993. Mon. Not. R. astron Soc., 261, 39. 